\title{
Bilateral serous retinal detachment in a case of eclampsia
}

\author{
Shiny Varghese ${ }^{1^{*}}$, Jacob Koshy $^{2}$, Kumkum Avasthi $^{1}$ \\ ${ }^{1}$ Department of Obstetrics and Gynaecology, Christian Medical College, Ludhiana, India \\ *Corresponding Author: shinyashishvarghese@gmail.com \\ ${ }^{2}$ Department of Ophthalmology, Christian Medical College, Ludhiana, India
}

Received 18 August 2011; revised 12 October 2011; accepted 24 November 2011

\begin{abstract}
Serous retinal detachment is a rare complication occurring in less than $1 \%$ of patients with eclampsia. We here report such a case. We strongly recommend the need for an eye check up in all patients of pregnancy induced hypertension especially in community based setup.
\end{abstract}

Keywords: Serous Retinal Detachment; Eclampsia; Pregnancy Induced Hypertension

\section{INTRODUCTION}

Visual disturbances are a common occurrence in cases of pre-eclampsia and are seen in $25 \%$ of women with pre-eclampsia and $50 \%$ of women with eclampsia [1]. This may be related to cerebral or ophthalmic vascular insufficiency secondary to complications of hypertension in pregnancy like optic neuropathy, serous retinal detachment and haemorrhagic or exudative maculopathy. Serous retinal detachment is a rare complication occurring in less than $1 \%$ of patients with eclampsia. We here report such a case.

\section{CASE REPORT}

A 32 years old lady $\mathrm{G}_{3} \mathrm{P}_{1+0}\left(\mathrm{~L}_{0}\right)$ presented to our hospital at 35 weeks of pregnancy with two episodes of tonic clonic seizures and an ultrasound showing an intrauterine fetal demise.

She had irregular antenatal checkups elsewhere. Her pregnancy was uneventful until fifteen days prior to admission when she started having episodic headache with high BP recordings. There was no history of any visual disturbances prior to admission. Twelve years ago she delivered a female baby, who died 3 days after birth. Two years ago she had a spontaneous abortion at two months gestation.

She had no previous high blood pressure recordings.

On Physical Examination the patient was drowsy. She had bilateral pitting pedal oedema. Blood Pressure was 150/100 mmHg. On abdominal examination uterus corresponded to 32 weeks gestation with a cephalic presentation and absent fetal heart sounds. Per vaginal examination revealed a Bishops score of 4 with absent membranes. Rest of her examination was normal.

Ophthalmic examination. Visual acuity was 2/60 and $6 / 60$ in the right and left eyes respectively. Anterior segment examinations of both eyes were normal. Fundus examination revealed cotton-wool spots, superficial haemorrhages, focal and generalized constriction of the arterioles suggestive of Grade 3 Hypertensive Retinopathy. Serous retinal detachments were seen in both eyes.

Investigations. Her hemogram revealed leucocytosis $\left(21,300 / \mathrm{mm}^{3}\right)$, thrombocytopenia $\left(47,000 / \mathrm{mm}^{3}\right)$ and a mildly deranged coagulation profile (APTT—37.5”/26"). Peripheral blood film revealed rare schistocytes. Renal parameters were deranged (blood urea-101 mg\%, serum creatinine-1.1 mg\%). Uric acid was $12.8 \mathrm{mg} \%$. Liver function tests revealed hypoalbuminemia and mild elevation of transaminases. Urine analysis showed significant protienuria. Ultrasound (B scan) examination of both eyes confirmed the diagnosis of serous retinal detachment (Figures 1 and 2).

Treatment. She was started on magnesium sulphate regime and Tab Labetolol 200 mg QID. She was transfused four units each of fresh frozen plasma and random donor platelets. Labour was then augumented under coverage of broad spectrum antibiotics. She delivered a macerated female baby weighing $1.7 \mathrm{~kg}$.

Post-partum. BP records remained very high upto 180/ $120 \mathrm{mmHg}$ and hence Tab Labetalol was replaced with Tab Monoxidine $0.2 \mathrm{mg}$ TDS, Tab Amlong $5 \mathrm{mg}$ BD and Atenolol $50 \mathrm{mg}$ OD. Her renal function tests done serially showed gradual improvement. She was discharged after control of hypertension on $7^{\text {th }}$ post partum day and was lost to follow up.

\section{DISCUSSION}

Serous retinal detachment as a complication of pree- 


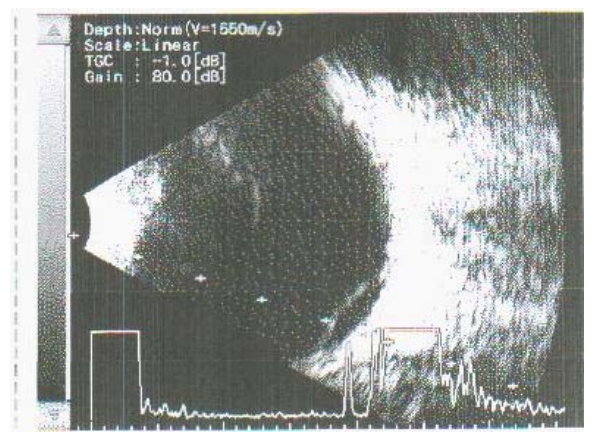

Figures 1. Ultrasonography of right and left eyes showing serous retinal detachments.

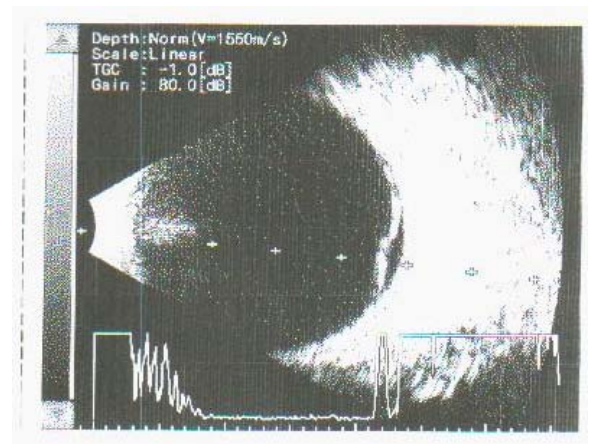

Figures 2. Ultrasonography of right and left eyes showing serous retinal detachments.

clampsia can occur at antepartum or postpartum period even in the presence of only mild hypertension and in absence of significant retinal vascular abnormalities. However it usually presents in patients with severe preeclampsia or eclampsia [2,3]. It has been reported in $1 \%$ to $2 \%$ of patients with severe preeclampsia and in $10 \%$ of patients with eclampsia [4]. Although retinal and choroidal vascular damage have been implicated in the pathogenesis, the exact mechanism of retinal detachment associated with preeclampsia is still unclear.

The most common abnormality seen in the visual system is spasm and narrowing of the retinal vessels reported in $70 \%$ of cases of toxemia [2]. Retinal hemorrhages and papilloedema in preeclampsia characterize patients with an acute, severe rise in blood pressure.
Temporary blindness associated with preeclampsia has been reported as originating from diffuse, generalized spasm of retinal arterioles, thrombosis of the central artery, papillophlebitis, cerebral spasm and edema [5]. Perhaps one of the best known, although rare, causes of loss of vision associated with preeclampsia is serous retinal detachment [3].

Choroidal ischaemia consequent to terminal arteriolar vasospasm affects the retinal pigment epithelium and leads to the breakdown of the blood retinal barrier, leakage of proteins and fluid from the choriocapillaris into the subretinal space and exudative retinal detachment [6]. A focal dome shaped elevation of the retina characterizes exudative retinal detachment. These retinal detachments usually settle down on its own once the eclampsia or systemic hypertension is controlled. In conclusion we strongly recommend the need for an eye check up in all patients of pregnancy induced hypertension especially in community based setup.

\section{REFERENCES}

[1] Sharma, S., Wuntakal, R., Anand, A., Sharma, T.K. and Downey, G. (2006) Pregnancy and the eye. The Obstetrician and Gynaecologist, 8, 141-146. doi:10.1576/toag.8.3.141.27248

[2] Sathish, S. and Arnold, J. J. (2000) Bilateral choroidal ischaemia and serous retinal detachment in pre-eclampsia. Clinical \& Experimental Ophthalmology, 28, 387-390. doi:10.1046/j.1442-9071.2000.00342.x

[3] Chatwani, A., Oyer, R. and Wong, S. (1989) Postpartum retinal detachment. Reproductive Medicine, 34, 842-844

[4] Lee, C., Hsu, T.Y., Ou, C.Y., Chang, S.Y. and Soong, Y.K. (1999) Retinal detachment in postpartum preeclampsia and eclampsia. Changgeng Yi Xue Za Zhi, 22, 520-524.

[5] Moodley, J., Pillay, M. and Pillay, R. (1985) Temporary blindness and eclampsia. South African Medical Journal, 68, 677-678.

[6] Landau, D., Seelenfreund, M.H., Tadmor, O., Silverstone, B.Z. and Diamant, Y. (1995) The effect of normal childbirth on eyes with abnormalities predisposing to rhegmatogenous retinal detachment. Graefe's Archive for Clinical and Experimental Ophthalmology, 233, 598-600. doi:10.1007/BF00404712 